\title{
Research on SLAM and Path Planning Based on ROS Robot
}

\author{
Linchen $\mathrm{Li}^{1}$, Xuehan $\mathrm{Li}^{2}$, Zhiguo Shi ${ }^{2}$, Genjing Chang ${ }^{3}$ \\ ${ }^{1}$ Beijing Polytechnic College \\ Beijing, China \\ 49131304@qq.com \\ ${ }^{2}$ University of Science and Technology Beijing \\ Beijing, China \\ g20188744@xs.ustb.edu.cn; szg@ustb.edu.cn \\ ${ }^{3}$ Beijing Ironman Technology Co. Ltd. \\ Beijing, China \\ bd@artrobot.com
}

\begin{abstract}
With the development of artificial intelligence technology, human-computer interaction has become the trend of robot research, navigation robots are widely used. Navigation robots autonomous navigation in an unknown environment, including perception of the environment, autonomous positioning and dynamic obstacle avoidance, etc. Among them, Simultaneous Localization and Mapping (SLAM) and path planning are prerequisites for achieving robot intelligent navigation. Aiming at problems such as low positioning accuracy and inability to accurately sense obstacles in 3D complex environments when using lidar. Firstly, based on the data characteristics of lidar and depth camera, the projection of point cloud collected by depth camera on 2D plane and lidar point cloud are fused by Bayes formula, then build an occupied grid map of the indoor complex environment. In addition, the traditional artificial potential field (APF) is easy to fall into the local optimal solution and oscillation of the potential field, and there may be collisions in the presence of dynamic obstacles. In the face of complex dynamic environments, it is difficult to plan a complete path. The velocity factor of obstacles is added to judge the movement trend of obstacles in the APF method, and a new repulsion function is introduced to overcome the local optimal trap and avoid the problem that obstacles near the target point lead to the target unreachable. Experiments show that the fusion raster map has higher precision than the single laser radar in the establishment of grid maps. In the aspect of path planning, the improved APF method can effectively solve the stochastic dynamic obstacles, and improve the navigation efficiency of robots.
\end{abstract}

Keywords: Navigation robot; SLAM; Bayes; Route plan; APF; Dynamic obstacle avoidance;

\section{Introduction}

Robot Operating System (ROS) provides a unified development platform for robots. It has the characteristics of compatibility, open source, portability, etc. It provides intelligent robots with the necessary operational support functions such as environment awareness and motion control[1]. In the field of intelligent robots, mapping and navigation is a core technology. The multi-sensor data fusion combined with intelligent control technology to develop an effective path planning method can make the robot more intelligent, and ROS provides the commonly used SLAM technology, which simplifies.

SLAM refers to the robot through the multi-sensor system in the process of moving to establish a description model of the surrounding environment, estimate their own position and trajectory, get the description of the robot's environment and the location information, and build the environment map[2]. The SLAM algorithm is mainly divided into Lidar SLAM and Visual SLAM. Lidar SLAM is the most stable method of location mapping, which mainly builds two-dimensional map. The Lidar SLAM algorithm is mostly based on Particle Filter (PF), Particle filter is simple and easy to implement, but it has the disadvantages of complex calculation and large vector dimension. It can only obtain plane information, not 3D feature information, which affects the global positioning. The Vision SLAM uses the depth camera to obtain the feature information of the map to construct, so as to construct the global consistent map. However, the image sensor is vulnerable to the influence of light changes, rapid movement, etc., which results in the low accuracy of the mapping and positioning.

By improving the performance of path planning, the efficiency of navigation robots in complex unknown environments can be effectively improved. The current path planning research is mainly divided into global path planning and local path planning. The global path planning method includes viewable method, grid method, Dijkstra algorithm and A* algorithm[3]; 
local path planning algorithm includes Fuzzy logic algorithm, genetic algorithm, RRT algorithm, ant colony algorithm, APF method. Compared with the complexity of most path optimization algorithms, the APF method judges the path according to the obstacles near the current location, and does not need to predict the complete path in advance. It has the characteristics of fast planning, smooth path and high real-time. However, the traditional APF method usually has the unreachable target and is easy to fall into the local optimal solution. The specific points are as follows: in the face of dynamic obstacles, it often causes obstacle avoidance failure; when the repulsion and gravity of the robot reach the balance, it will fall into the local optimal solution; when there are obstacles around the target point, the repulsion force of the robot near the target point is greater than the gravity, resulting in local vibration, resulting in the inability to reach the target point.

In response to the above problems, researchers have conducted research: Grisetti proposes a laser SLAM algorithm Gmapping based on RBPF-SLAM, which adds the lidar data to the proposed distribution and improves the efficiency of the algorithm[4]. Zhang proposes a laser radar and visual fusion mapping algorithm with high robustness and low drift to improve its accuracy[5]. Guo combines the APF method with the navigation potential field method to solve the problem of unreachable target[6].

This paper is based on ROS mobile robot, firstly, aiming at the limitation of localization and mapping of ladar SLAM Algorithm in complex environment limited to 2D scene and unable to obtain global information, the 3D point cloud constructed with visual slam contains rich 3D feature information. The projection of 3D point cloud on 2D plane is fused with 2D lidar data to build a global occupier grid map based on Bayesian formula. At the same time, in view of the problems existing in the process of path navigation of robot based on APF method, the improved repulsion function is introduced again, so that the repulsion function is not only affected by the distance between the robot and the obstacle, but also affected by the relative position of the target point and the robot, so as to avoid the problem of inaccessibility of the target and overcome the local optimal solution. Considering the relative velocity of obstacles and robots, the velocity coefficient is introduced to judge the relative motion trend, and the gravity and repulsion functions under the relative velocity are calculated to avoid collision between robots and dynamic obstacles. Finally, based on the ROS robot platform, the lidar and the depth camera are fused to establish a grid map containing 3D information and MATLAB is used to simulate the improved APFalgorithm and verify its feasibility.

\section{Map fusion based on lidar and depth camera}

The process of robot SLAM is to control the robot to reach a pose, and then to observe the robot's position in the scene. Lidar obtains map scene information by building occupation grid map. Grid map is a discrete representation of the environment, which represents the map as a series of grid units of equal size, and is divided into rows and columns. Each grid is given a value to represent the grid state, which is used to represent the probability that the grid is occupied or not occupied by obstacles. Grid map is easy to create and maintain, but in large-scale environment, with the expansion of local map, its calculation will increase, so with the help of depth camera to obtain global and three-dimensional spatial information, the accuracy of map creation and location can be improved by multi-sensor system.

For the single occupied grid map, Bayesian formula is used to update the map state: $O_{i}$ represents the grid occupied by the i-th grid cell at time $t, \bar{O}_{i}$ indicates unoccupied, $E$ indicates the presence of an obstacle, $P(E)$ represents the prior probability of an obstacle, $P(\bar{E})$ represents the prior probability that an obstacle does not exist, $P\left(O_{i} \mid E\right), P\left(O_{i} \mid \bar{E}\right)$ represents the sensor observation model and update the grid map model with the observations of the conditional probabilities $P\left(E \mid O_{i}\right)$, as shown in Eqs. (1):

$$
P\left(E \mid O_{i}\right)=\frac{P\left(O_{i} \mid E\right) P(E)}{P\left(O_{i} \mid E\right) P(E)+P\left(O_{i} \mid \bar{E}\right) P(\bar{E})}
$$

For the fusion of lidar and depth camera, Elkan proposes to update the multi-sensor fusion observation data raster graph using Bayesian recursion formula[7,8], as shown in Fig. 1. 


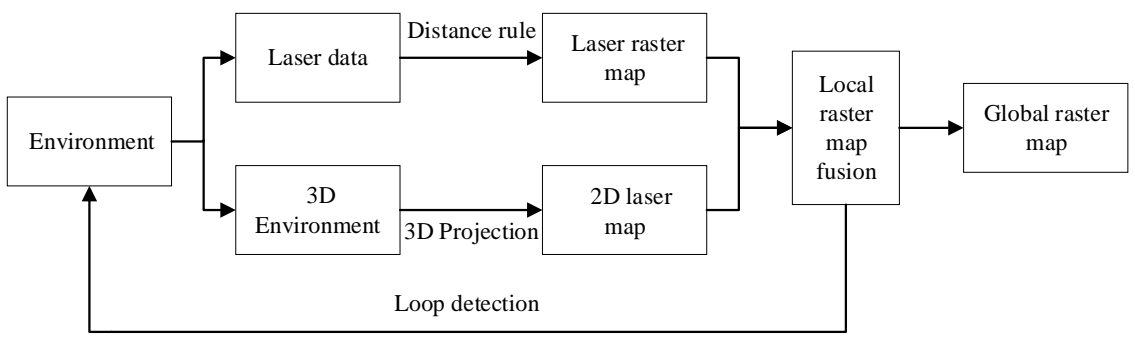

Fig. 1: Multi-sensor fusion process.

For the current raster state of the two sensors, the fusion rule as shown in Eqs. (2), $P(i)$ indicates that the merged grid is occupied, $P_{l}(i)$ indicates that the grid detected by lidar is occupied, $P_{D}(i)$ indicates that the grid detected by depth camera is occupied.

$$
P(i)=P_{l}(i) \cup P_{D}(i)
$$

After the local raster map is known, the global raster map is drawn by combining the local maps at different moments, as shown in Eqs. (3), $Z_{t}$ represents the observed value status (occupied, idle) of the local map at the time $t$, similarly $Z_{t+1}$; $P\left(O_{i} \mid Z_{t+1}\right)$ represents the probability that the i-th grid is occupied by the obstacle after the observation of the $t+1$ time; $P\left(O_{i} \mid Z_{t}\right)$ represents the probability that the $\mathrm{i}$-th grid is occupied by the obstacle after the observation at the time $t$ is merged.; $P_{E}\left(O_{i} \mid Z_{t+1}\right)$ represents the probability that the $\mathrm{i}$-th grid judged by the observation at the current $t+1$ time is occupied by the obstacle.

$$
P\left(O_{i} \mid z_{t+1}\right)=\left[1+\frac{1-P_{E}\left(O_{i} \mid Z_{t+1}\right)}{P_{E}\left(O_{i} \mid Z_{t+1}\right)} \frac{1-P\left(O_{i} \mid Z_{t}\right)}{P\left(O_{i} \mid Z_{t}\right)}\right]^{-1}
$$

\section{APF}

\subsection{Traditional APF}

APF is a virtual force method proposed by Khatib, which designs the motion space of the robot in the surrounding environment as an abstract virtual force field[9]. The target point produces the gravitational potential field $\vec{U}_{a t t}$ to the robot, calculates the negative gradient to obtain its gravitational force $\vec{F}_{a t t}$, the obstacle produces the repulsive potential field $\vec{U}_{\text {rep }}$ to the robot, calculates the negative gradient to obtain $\vec{F}_{r e p}$, and finally controls the robot movement through the resultant force $\vec{F}_{\text {total }}[10]$, as shown in Fig. 2.

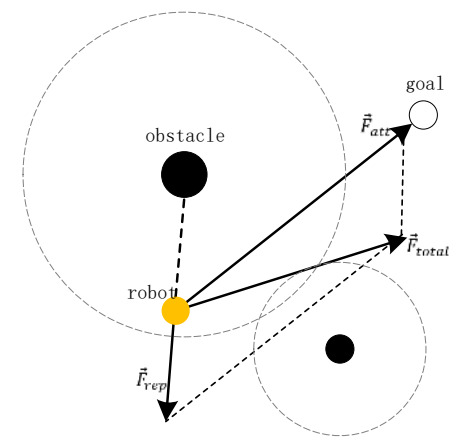

Fig.2 APF diagram

Let the robot move in a two-dimensional space, which includes the target point and several obstacles. The position of the robot in the space is expressed as $q(x, y)^{T}$, and the gravitation function of the target point to the robot as shown in Eqs. (4), find the negative gradient to get the gravity of the robot as shown in Eqs. (5); The repulsion function of the obstacle and the robot as shown in Eqs. (6), find the negative gradient to get the gravity of the robot as shown in Eqs. (7); For multiple obstacles, the resultant force of the robot at position $q$ as shown in Eqs. (8). 


$$
\begin{gathered}
\vec{U}_{a t t}(q)=\frac{1}{2} k_{a t t} \rho^{2}\left(q, q_{\text {goal }}\right) \\
\vec{F}_{a t t}(q)=-\nabla \vec{U}_{\text {att }}(q)=-k_{\text {att }} \rho\left(q, q_{\text {goal }}\right) \nabla \rho\left(q, q_{\text {goal }}\right) \\
\vec{U}_{r e p}(q)=\left\{\begin{array}{l}
\frac{1}{2} k_{\text {rep }}\left(\frac{1}{\rho\left(q, q_{o b s}\right)}-\frac{1}{\rho_{0}}\right)^{2}, \rho\left(q, q_{o b s}\right) \leq \rho_{0} \\
0 \quad, \rho\left(q, q_{o b s}\right)>\rho_{0}
\end{array}\right. \\
\vec{F}_{r e p}(q)=-\nabla \vec{U}_{r e p}(q)=\left\{\begin{array}{l}
\frac{k_{r e p}}{\rho^{2}\left(q, q_{o b s}\right)}\left(\frac{1}{\rho\left(q, q_{o b s}\right)}-\frac{1}{\rho_{0}}\right) \nabla \rho\left(q, q_{o b s}\right), \rho\left(q, q_{o b s}\right) \leq \rho_{0} \\
0 \quad, \rho\left(q, q_{o b s}\right)>\rho_{0} \\
\vec{F}_{\text {total }}(q)=\vec{F}_{a t t}(q)+\sum_{i} \vec{F}_{r e p}(q) \quad
\end{array}\right.
\end{gathered}
$$

$k_{a t t}$ is the gravitational gain coefficient and $\rho\left(q, q_{\text {goal }}\right)$ is the relative distance between the robot and the target point, $\rho\left(q, q_{\text {goal }}\right)=\left|q-q_{\text {goal }}\right| ; k_{\text {rep }}$ is the repulsion gain coefficient, $\rho\left(q, q_{o b s}\right)$ is the relative distance between the robot and the obstacle, $\rho\left(q, q_{o b s}\right)=\left|q-q_{o b s}\right|, \rho_{0}$ is the influence distance of the obstacle.

\subsection{Improved APF}

According to the APF method, the robot's moving direction is determined by the combined force of the target point's gravity and the obstacle's repulsion. It is known from Eqs. (5) and (7) that the gravity of the robot is directly proportional to the distance to the target point, and the repulsion force of the robot is inversely proportional to the distance to the obstacle. When the target point is close to the obstacle, the gravity of the robot will gradually reduce, and the repulsion force will gradually increase, so the robot will move away from the target point when the repulsion force is greater than the gravity, resulting in vibration[11].

In order to solve the problem that the target is not reachable the improved repulsion function $\rho^{n}\left(q, q_{\text {goal }}\right), n>1$ is introduced into the APF, and the relative distance $\rho\left(q, q_{\text {goal }}\right)$ between the robot and the target point is added to the obstacle repulsion potential field as an influence factor. When the robot is close to the target point, the repulsion force will gradually reduce to 0 to avoid the robot vibration near the target point. The improved repulsion potential field function as shown in Eqs. (9), When $\rho\left(q, q_{\text {obs }}\right) \leq \rho_{0}$, the negative gradient is obtained as shown in Eqs. (10).

$$
\begin{aligned}
& \vec{U}_{\text {repq }}(q)= \begin{cases}\frac{1}{2} k_{\text {rep }}\left(\frac{1}{\rho\left(q, q_{o b s}\right)}-\frac{1}{\rho_{0}}\right)^{2} \rho^{n}\left(q, q_{\text {goal }}\right), \rho\left(q, q_{o b s}\right) \leq \rho_{0} \\
0 & , \rho\left(q, q_{o b s}\right)>\rho_{0}\end{cases} \\
& \vec{F}_{\text {repq }}(q)=-\nabla \vec{U}_{r e p}(q)=\vec{F}_{1}+\vec{F}_{2} \\
& =\frac{k_{\text {rep }}}{\rho^{2}\left(q, q_{o b s}\right)}\left(\frac{1}{\rho\left(q, q_{o b s}\right)}-\frac{1}{\rho_{0}}\right) \rho^{n}\left(q, q_{\text {goal }}\right) \nabla \rho\left(q, q_{o b s}\right) \\
& +\frac{n}{2} k_{\text {rep }}\left(\frac{1}{\rho\left(p-p_{o b s}\right)}-\frac{1}{d_{0}}\right)^{2} \rho^{n-1}\left(q, q_{\text {goal }}\right) \nabla \rho\left(q, q_{\text {goal }}\right)
\end{aligned}
$$

By definition, $\nabla \rho\left(q, q_{\text {goal }}\right)$ and $\nabla \rho\left(q, q_{o b s}\right)$ is constant. $\vec{F}_{r e p q}(q)$ is the repulsion model of the robot in the modified APF, where the force is decomposed into forces $\vec{F}_{1}$ and $\vec{F}_{2}$ in two directions, where $\vec{F}_{1}$ is the force from the obstacle to the robot, The robot generates a repulsive force, and $\vec{F}_{2}$ is the force that points from the robot to the target point, generating gravity to the robot. $n$ is the exponential coefficient. When $n>1, \vec{F}_{1}$ will gradually approach 0 as $\rho\left(q, q_{\text {goal }}\right)$ decreases. Analysis of Eqs. (10):

$$
\begin{array}{r}
\lim _{\rho\left(q, q_{\text {goal }}\right) \rightarrow 0} \vec{F}_{1}=\lim _{\rho\left(q, q_{\text {goal }}\right) \rightarrow 0} \frac{k_{\text {rep }}}{\rho^{2}\left(q, q_{\text {obs }}\right)}\left(\frac{1}{\rho\left(q, q_{o b s}\right)}-\frac{1}{\rho_{0}}\right) \rho^{n}\left(q, q_{\text {goal }}\right) \nabla \rho\left(q, q_{\text {obs }}\right)=0 \\
\lim _{\rho\left(q, q_{\text {goal }}\right) \rightarrow 0} \vec{F}_{2}=\lim _{\rho\left(q, q_{\text {goal }}\right) \rightarrow 0} \frac{n}{2} k_{r e p}\left(\frac{1}{\rho\left(p-p_{\text {obs }}\right)}-\frac{1}{d_{0}}\right)^{2} \rho^{n-1}\left(q, q_{\text {goal }}\right) \nabla \rho\left(q, q_{\text {goal }}\right)=0
\end{array}
$$

When the robot is close to the target point, the repulsion force of the obstacle to the robot approaches to 0 , and the attraction force of the target point to the robot also approaches to 0 . Robot is close to the target under the action of $\vec{F}_{\text {att }}(q)$.

In addition, when the traditional APF is faced with dynamic obstacles, if the moving speed of obstacles is too large, it will often cause collision. Therefore, the APF method adds the speed term of obstacles, calculates the relative speed of robots 
and obstacles, and estimates the movement trend. Same as equation (12), establish the velocity potential field model as shown in Eqs. (13):

$$
\vec{U}_{\text {repv }}(v)= \begin{cases}\frac{1}{2} k_{r e p v} f^{2}\left(v, v_{o b s}\right) & , f\left(v, v_{o b s}\right) \geq 0 \\ 0 & , f\left(v, v_{o b s}\right)<0\end{cases}
$$

$\vec{U}_{r e p v}(v)$ is the established repulsion velocity potential field, $k_{r e p v}$ is the velocity coefficient, $f\left(v, v_{o b s}\right)$ is the relative velocity between the robot and the obstacle, $f\left(v, v_{o b s}\right)=v-v_{o b s}$, and $f\left(v, v_{o b s}\right) \geq 0$ indicates the Relatively close, there is a risk of collision, calculate the negative gradient to obtain the repulsion model of velocity when $f\left(v, v_{o b s}\right) \geq 0$, as shown in Eqs. (14).

$$
\vec{F}_{\text {repv }}(v)=-\nabla \vec{U}_{\text {repv }}(v)=-k_{\text {repv }} f\left(v, v_{\text {obs }}\right)
$$

The comprehensive repulsion function $\vec{F}_{r e p}(q, v)$ can be obtained from Eqs. (10) and (14), as shown in Eqs. (15).

$$
\vec{F}_{r e p}(q, v)=\left\{\begin{array}{cc}
\vec{F}_{r e p q}(q)+\vec{F}_{r e p v}(v) & \rho\left(q, q_{o b s}\right) \leq \rho_{0} \text { 且 } f\left(v, v_{o b s}\right) \geq 0 \\
\vec{F}_{r e p q}(q) & \rho\left(q, q_{o b s}\right) \leq \rho_{0} \text { 且 } f\left(v, v_{o b s}\right) \leqslant 0 \\
0 & \rho\left(q, q_{o b s}\right)>\rho_{0}
\end{array}\right.
$$

Aiming at the problem that the resultant force of gravity and repulsive force is 0 in the process of robot movement, increasing the velocity term of the obstacle can effectively avoid the situation. The velocity repulsive force of the dynamic obstacle in the robot motion space will fall into the optimal solution. The robot generates a state disturbance that helps the robot get out of the optimal trap.

\section{Simulation Results Analysis}

\subsection{Simulation Analysis of Lidar and Depth Camera Fusion}

Set up a mobile robot experimental platform. The robot running system is Ubuntu 16.04, ROS Kinetic, processor i5$6200 \mathrm{U}$, memory is $4 \mathrm{G}$, hard disk space is $128 \mathrm{G}$; Remote control terminal uses personal PC, system is Ubuntu 16.04, ROS Kinetic, processor i5- 6500, the memory is $8 \mathrm{G}$, and the hard disk space is $360 \mathrm{G}$. According to the ROS robot operating system to build the modules required for the experiment, the lidar uses the YDLIDAR F4PRO lidar developed by EAI. The depth camera adopts Kinect V2 preview version, and adopts TOF method to obtain the image depth information through the time returned from the reflected infrared ray.

Construct the experimental scene and control the robot to move in the experimental environment. First, use the lidar as the sensor. The remote PC views the grid map effect obtained through the visualization platform Rviz in ROS, as shown in Fig. 3. Then turn on the depth camera, combine the lidar to obtain the field environment, control the robot to move in the same environment, and view the fused grid map through Rviz, as shown in Fig. 4. It can be seen from the figure that the grid map constructed after fusion has higher precision and better effect than the grid map obtained only by lidar; especially for the obstacles with shorter position than the robot lidar, the lidar can not get its information accurately, and the depth camera can detect the area lower than its position, effectively making up for the laser lack of radar.

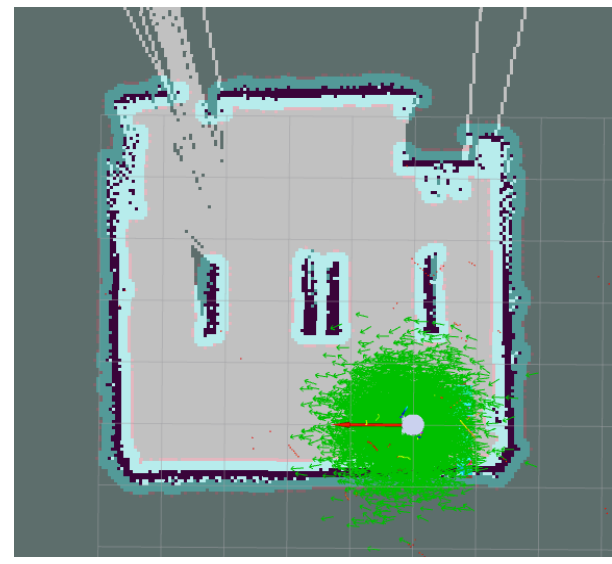

Fig.3 Grid map using lidar

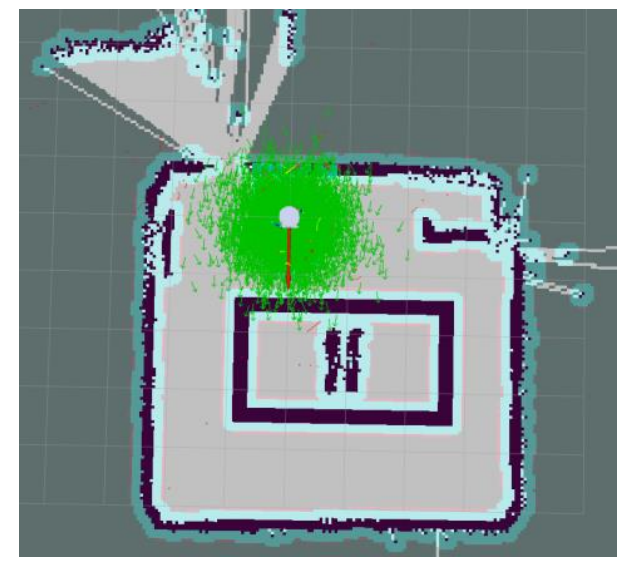

Fig.4 Grid map of lidar and depth camera 


\subsection{Improved APF Simulation Analysis}

Simulate the moving path of the modified APF method, set the starting coordinates $(0,0)$, the target coordinates $(10,10)$, the gravitational coefficient $k_{a t t p}=15$, the repulsion coefficient $k_{\text {repp }}=2$, the robot step size is 0.2 , the obstacle step The length is 0.15 , the speed coefficient $k_{\text {repv }}=0$, and the obstacle influence radius is uniformly defined as $\rho_{0}=3$; the process of simulating the robot to avoid the dynamic obstacle reaching the target point, as shown in Fig. 5, includes dynamic obstacles and static obstacles.
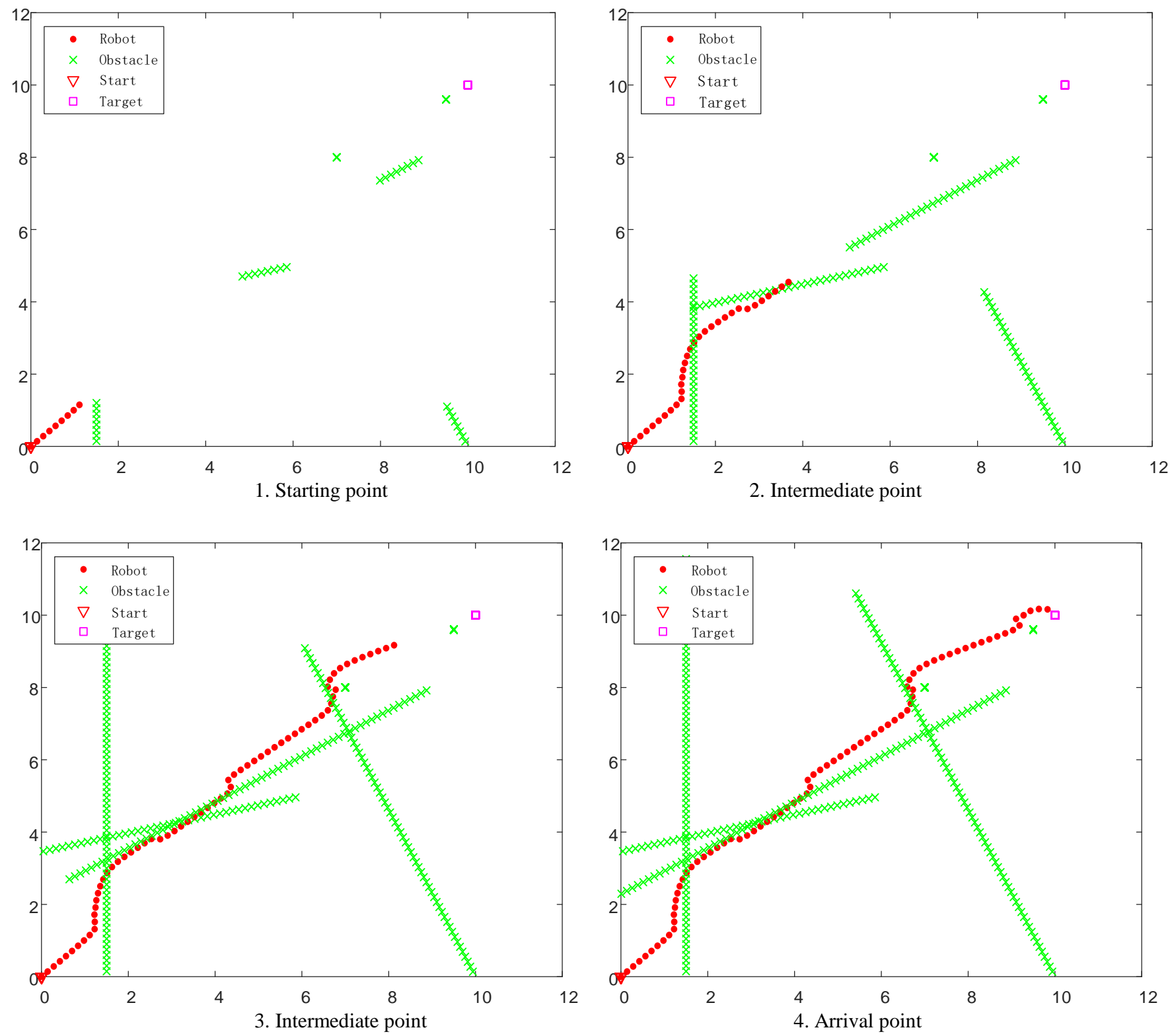

Fig.5 Robot dynamic path planning

The traditional APF method and the improved APF method are respectively used to simulate and verify the problem that the robot cannot reach the target point. Static obstacles are used in the verification process, as shown in Fig. 6 and Fig. 7. Through comparison, it can be found that when there are obstacles near the target point, using the traditional APF method, the robot will generate vibration near the target point and cannot reach the target point; using the improved APF method, the robot can reach the target point accurately. 


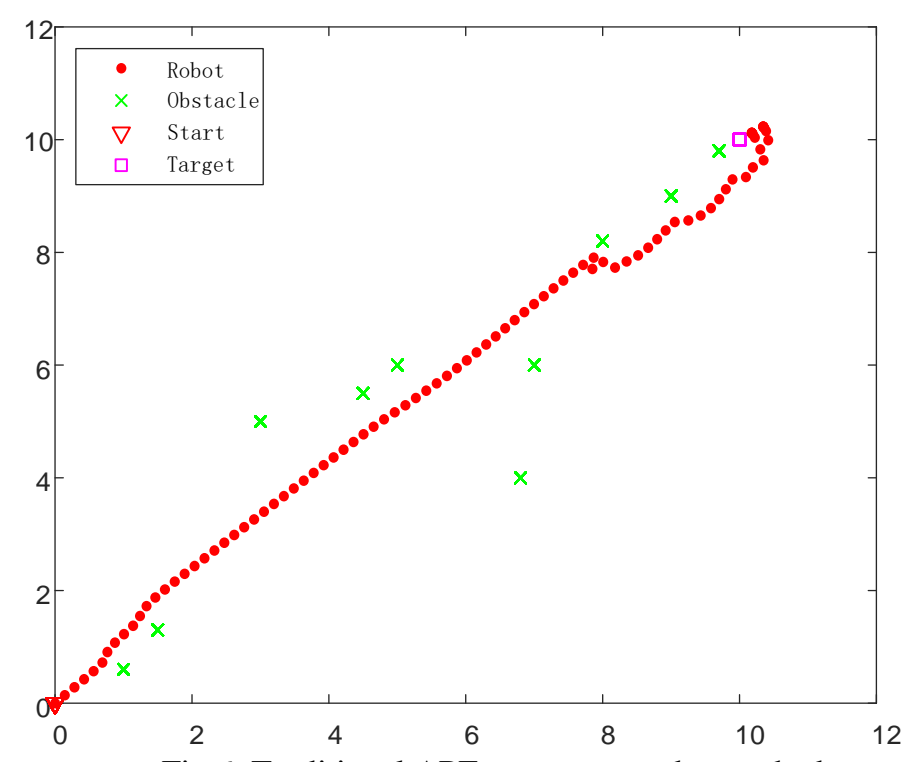

Fig.6 Traditional APF target cannot be reached

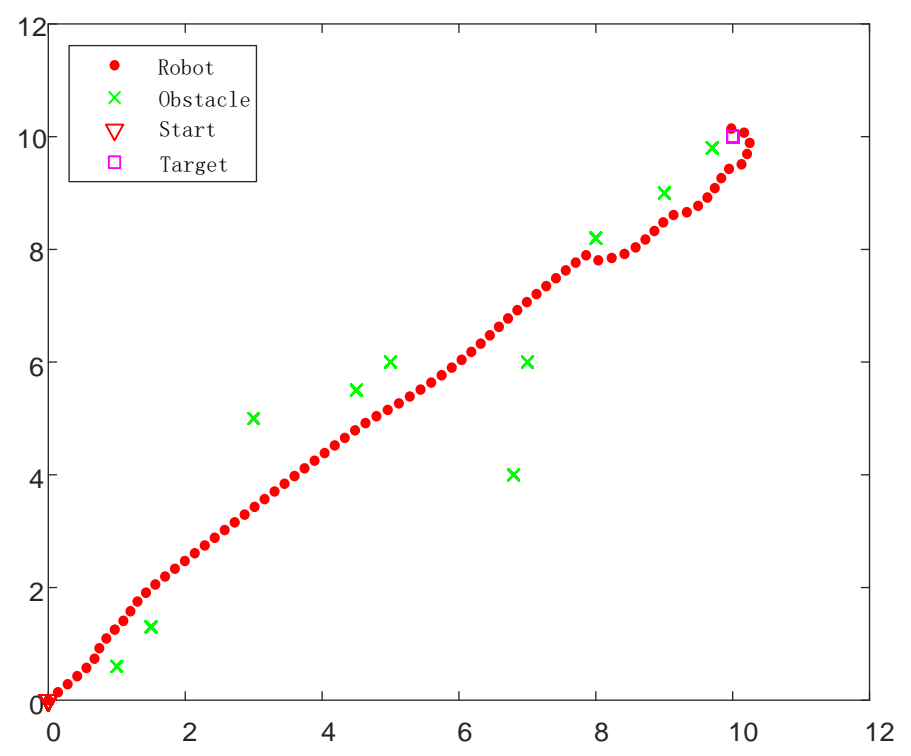

Fig.7 Improve APF target can be reached

For the problem of dynamic obstacles, based on the traditional APF method, the moving path is shown in Fig. 8. It can be seen from the figure hat when the distance between the robot and the dynamic obstacles is relatively close, the moving direction will have a sudden change. This is because the robot is subject to too much traction, and only when the distance between the robot and the obstacles is very close can the repulsion force resist the influence of gravity; The moving path of the APF method with the velocity term is shown in Fig. 9. Through comparison, it is found that the traditional method needs 90 steps to move to the target point, and the improved method only needs 78 steps, which reduces the number of path points by $13.3 \%$.
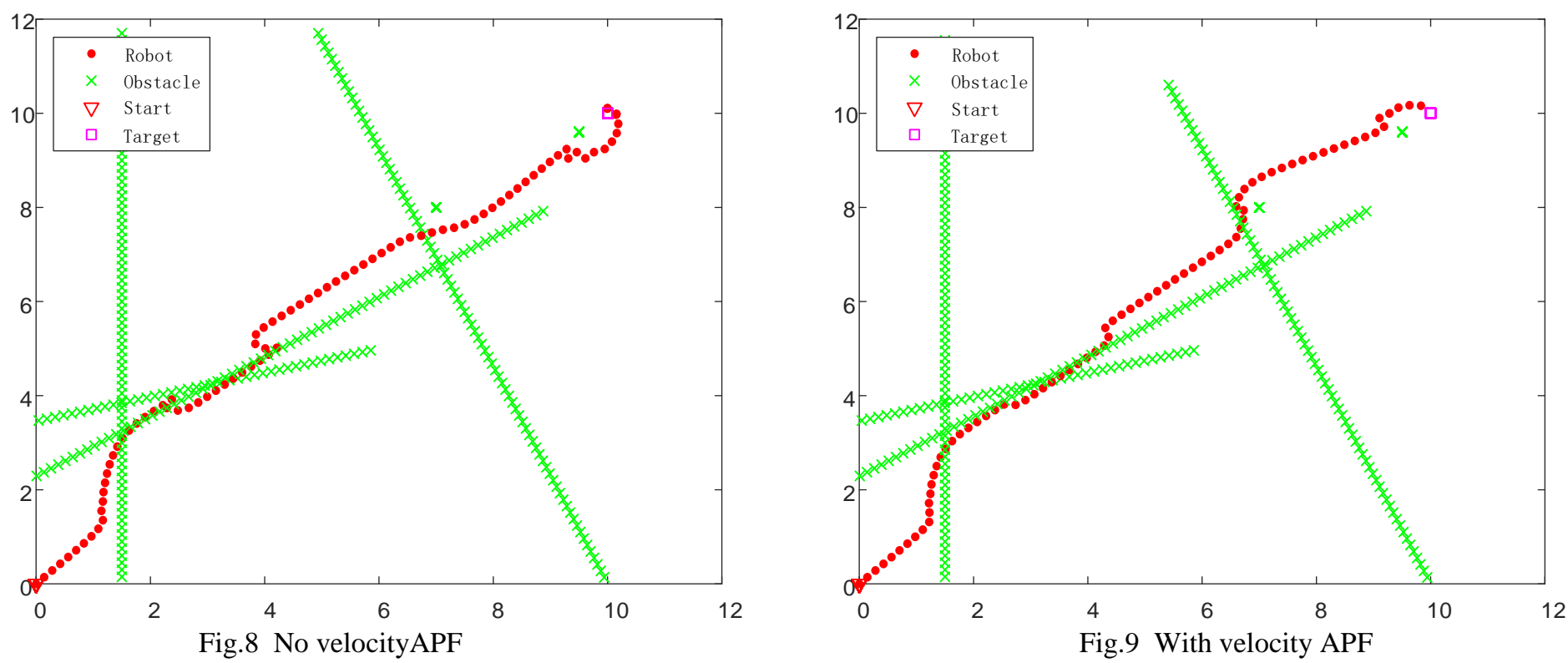

Compared with the traditional APF method, the improved APF method has better performance and higher efficiency, which can make timely response to dynamic obstacles and prevent them from colliding with each other; at the same time, it also has a good improvement for the problem of the inaccessibility of the target, avoiding the vibration of the robot.

Finally, according to the dynamic 2D space that has been set, the genetic algorithm, RRT algorithm and improved APF method are simulated and compared, and their performance are compared, as shown in Table 2, the number and accuracy of 
several algorithms in path planning are listed. Through the comparison, we can see that the improved APF method has higher precision and can better complete the path planning.

Table 2 Path planning performance comparison

\begin{tabular}{ccccc}
\hline Method & Genetic Algorithm & RRT & Traditional APF & Improved APF \\
\hline Path points & 81 & 78 & 90 & 78 \\
Accuracy & $87.30 \%$ & $89.28 \%$ & $84.28 \%$ & $90.52 \%$ \\
\hline
\end{tabular}

\section{Conclusion}

In the process of creating SLAM map of ROS robot, in the face of complex scenes, it is impossible to accurately acquire the environment by relying solely on lidar, especially for the obstacles that are not in the same plane as lidar. Therefore, multi-sensor fusion is used to obtain three-dimensional scene information by combining depth camera, and data fusion between lidar and depth camera is established based on Bayesian method Global grid map. Finally, the experimental platform of ROS robot is built, and the feasibility of this method is verified by simulation. The results show that the fused grid map has better global and accuracy.

In the process of path planning, the traditional APF has the problems of inaccessibility and inability to deal with dynamic obstacles. Based on these two main problems, a new repulsion function is defined to make the repulsion of obstacles affected by the distance between the robot and the target point; For dynamic obstacles, the velocity potential field is added to make the robot predict the path of obstacles in advance. Based on the improved APF method, the simulation is designed in the MATLAB environment. Through the simulation results, we can see that the improved algorithm has higher efficiency and performance. The algorithm meets the requirements of robot obstacle avoidance path planning.

\section{Acknowledgments}

This work was supported by National key R \& D program funded projects(No.2018YFC0810601) and National Natural Science Foundation of China(No.61977005).

\section{References}

[1] D. B. Licea, D. McLernon, M. Ghogho, "Mobile Robot Path Planners With Memory for Mobility Diversity Algorithms," in IEEE Transactions on Robotics, vol. 33, no. 2, pp. 419-431, April 2017.

[2] M. W. M. G. Dissanayake, P. Newman, S. Clark, H. F. Durrant-Whyte, M. Csorba, "A solution to the simultaneous localization and map building (SLAM) problem," in IEEE Transactions on Robotics and Automation, vol. 17, no. 3, pp. 229-241, June 2001.

[3] E. W. Dijkstra, "A note on two problems in connexion with graphs," Numerische Mathematik,1959, vol. 1, no. 1, pp. 269-271.

[4] G. Grisetti, C. Stachniss, W. Burgard, "Improved Techniques for Grid Mapping With Rao-Blackwellized Particle Filters," in IEEE Transactions on Robotics, vol. 23, no. 1, pp. 34-46, Feb. 2007.

[5] J. Zhang, S. Singh, "Visual-lidar odometry and mapping: low-drift, robust, and fast," 2015 IEEE International Conference on Robotics and Automation (ICRA), Seattle, WA, 2015, pp. 2174-2181.

[6] J. Guo, Y. Gao, G. Cui, "Path Planning of Mobile Robot Based on Improved Potential Field," Information Technology Journal, 2013, vol. 12, no. 11, pp. 2188-2194.

[7] C. Elkan, "The Foundations of Cost-Sensitive Learning," Proceedings of the Seventeenth International Conference on Artificial Intelligence, Seattle, 2001, pp. 4-10.

[8] S. Lomax, S. Vadera, "An empirical comparison of cost-sensitive decision tree induction algorithms," Expert Systems, 2011, vol. 28, no. 3, pp.227-268.

[9] O. Khatib, "Real-time obstacle avoidance for manipulators and mobile robots," in Proceedings of International Conference Robotics and Automation, St. Louis, Mo, USA, pp. 500-505, March 1985.

[10] W. B. Chen, X. B. Wu, Y. Lu, "An Improved Path Planning Method Based on Artificial Potential Field for a Mobile Robot," Cybernetics and Information Technologies, vol. 15, no. 2, pp. 181, 2015.

[11] X. X. Liang, C. Y. Liu, X. L. Song, C. M. Hao, "Research on mobile robot path planning in dynamic environment," IEEE 2017 Chinese Automation Congress, Jinan, 2017, pp. 3890-3894. 